\title{
Reminder: Similar and differential effects in amnesic and weakly trained rats
}

\author{
TERRY L. DeVIETTI and DARLENE A. HAYNES \\ Central Washington State College, Ellensburg, Washington 98926
}

\begin{abstract}
Two experiments investigated the effectiveness of reminder treatments in potentiating memory in subjects given either strong fear conditioning and ECS or weak fear conditioning. In Experiment I, reminder increased memory in both groups. Experiment II substantiated this finding under somewhat different conditions and also showed that, in one reminder condition, increased memory was obtained in subjects fear conditioned and given ECS while no effect was noted in subjects given weak fear conditioning. The finding of differential modification of memory with reminder in these two groups suggests that a previous interpretation, based on the usual finding of parallel modification with reminder in these groups, may be incorrect. Moreover, these data suggest that the major effect of ECS is on memory retrieval processes.
\end{abstract}

In animals, memory, originally suppressed by a posttraining trial electroconvulsive shock (ECS), often recovers when conditions reminiscent of training are interpolated between training ECS and the test (e.g., Koppenaal, Jagoda, \& Cruce, 1967; Lewis, Misanin, \& Miller, 1968). Some have viewed this so called "reminder effect" as supporting the notion that ECS produces amnesia by interfering with memory retrieval processes (DeVietti \& Hopfer, 1974b; Lewis, 1969; Miller \& Springer, 1973, 1974). In this view, reminder releases for behavioral expression a formerly strong but unretrievable memory. Others, who hold to the traditional view that ECS disrupts memory consolidation, have interpreted the reminder data quite differently (Cherkin, 1972; Gold, Haycock, Macri, \& McGaugh, 1973; Gold \& King, 1974; Haycock, Gold, Macri, \& McGaugh, 1973; Kesner \& Conner, 1974; Mah \& Albert, 1973). This latter position holds that ECS, under some conditions, is not completely effective in disrupting memory consolidation. Reminder, in this formulation, provides a weak training experience which generalizes to and summates with the memory remnant surviving ECS. This generalization hypothesis received a measure of support recently (Gold et al., 1973; Haycock et al., 1973) when it was demonstrated that reminder increased retention both in subjects given strong training in a passive avoidance task followed by amnesic treatment (direct stimulation of the cortex and ECS, respectively) and in subjects given weak training but no amnesic treatment. This finding could suggest that reminder interacts with weak memories, however effected, to increase retention.

This research was supported in part by Grant GB-38790 from the National Science Foundation. Appreciation is extended to Craig E. Bucy for his aid in the collection of the data. Requests for reprints should be sent to Terry L. DeVietti, Department of Psychology, Central Washington State College, Ellensburg, Washington 98926.

\section{EXPERIMENT I}

The present experiment sought to determine if the parallel increase in retention following reminder in subjects given weak training and subjects given strong training and amnesic treatment shown in passive avoidance tasks (Gold et al., 1973; Haycock et al., 1973) could be replicated in a one-trial fear conditioning paradigm.

\section{Method}

Subjects and apparatus. Subjects were 98 male Long-Evans rats from the Central Washington State Psychology Department animal colony and aged 90-120 days at the beginning of the experiment. The apparatus has been described in detail elsewhere (DeVietti \& Larson, 1971). Two identical chambers were used for fear conditioning and testing. They measured $20 \times 30 \mathrm{~cm}$ and were $30 \mathrm{~cm}$ high. The sides and top were Plexiglas, while the back and front were aluminum painted flat gray. The grid floor was $.64-\mathrm{cm}$-diam stainless steel rods spaced $1.90 \mathrm{~cm}$ apart. A small hole just off center in the front wall allowed the insertion of a drinking tube with the orifice located approximately $5 \mathrm{~cm}$ into the chamber and $5 \mathrm{~cm}$ above the grid floor. A drinkometer circuit was formed between the water in the drinking tube and the grid floor. A $10-\mathrm{cm}$ loudspeaker for delivery of the conditioned stimulus $(15-\mathrm{sec} 1,200-\mathrm{Hz} 90-\mathrm{dB}$ tone from a Heathkit tone generator) was positioned at floor level behind the rear wall of each chamber. Both chambers were individually enclosed in lighted fan-ventilated sound-attenuation boxes. A chamber used to administer noncontingent treatments had walls constructed entirely of Plexiglas ( $21 \times 56 \times 21 \mathrm{~cm}$ high) with a grid floor the same size and spacing as in the training-testing chambers. This apparatus was housed in a different room from the training-testing chambers. Scrambled footshock, delivered through the grid floor of the conditioning chamber was either "strong" (1.6 mA; $3 \mathrm{sec} ; 60 \mathrm{~Hz} ; 330 \mathrm{~V} \mathrm{rms})$ or "weak" $(1.0 \mathrm{~mA}$; $1 \mathrm{sec} ; 60 \mathrm{~Hz}$; $206 \mathrm{~V} \mathrm{rms}$ ). Strong footshock was also administered in the noncontingent treatment chamber. Another chamber, a grid box, measured $20 \times 23 \times 20 \mathrm{~cm}$ high and was constructed of Plexiglas with the front and rear walls of anodized aluminum. It was used to administer a scrambled weak reminder footshock $(.6 \mathrm{~mA} ; 3 \mathrm{sec} ; 60 \mathrm{~Hz} ; 124 \mathrm{~V} \mathrm{rms}) 121 \mathrm{~h}$ after fear conditioning or noncontingent treatment. This apparatus was housed in yet another room. An ECS apparatus 
Table 1

Treatments and Retention Performance (Mean Log Drink Latencies \pm 1 Standard Error) of Groups in Experiment I

\begin{tabular}{lccccc} 
& \multicolumn{4}{c}{ Reminder } \\
\cline { 3 - 5 } Group & $\mathrm{N}$ & $\begin{array}{c}\text { Training } \\
\text { Cues }\end{array}$ & $\begin{array}{c}\text { Foot- } \\
\text { shock }\end{array}$ & \multicolumn{1}{c}{ Test } \\
\hline TSFS-WR & 12 & Yes & Yes & 2.71 & $( \pm .04)$ \\
TSFS-NR & 13 & No & No & 2.49 & $( \pm .10)$ \\
TWFS-WR & 12 & Yes & Yes & 2.50 & $( \pm .08)$ \\
TWFS-NR & 13 & No & No & 2.16 & $( \pm .08)$ \\
TSFS/ECS-WR & 12 & Yes & Yes & 2.24 & $( \pm .13)$ \\
TSFS/ECS-NR & 12 & No & No & 1.85 & $( \pm .12)$ \\
NCSFS/ECS-WR & 12 & Yes & Yes & 1.68 & $( \pm .20)$ \\
NCSFS/ECS-NR & 12 & No & No & 1.62 & $( \pm .16)$ \\
\hline
\end{tabular}

Note-Descriptions of the treatment groups may be found in the text. Abbreviations of reminder conditions are: $W R=$ weak reminder, $N R=$ no reminder.

(Hayes, 1948) was set to deliver a 92-mA shock (200 msec; $60 \mathrm{~Hz} ; 1,840 \mathrm{~V} \mathrm{rms}$ ) to the ears of the rat through wire clips fashioned to interlock with baby clothes snaps (Prims No. 0) previously fitted to each ear.

Procedure. The subjects were fitted with ear snaps and then individually housed. Food and water were provided ad lib throughout the experiment except that water was withheld $24 \mathrm{~h}$ prior to drink training and testing (see below). On the day following the start of individual housing, each rat was handled for $5 \mathrm{~min}$. During the next 4 days, the subjects were given experience with the various aspects of the experimental situation according to the following schedule. On the first day, each rat was placed into one of the two training-testing chambers and left there for $10 \mathrm{~min}$. During this interval, water was removed from the home cage. The following day, each rat was taken to the chamber, its nose was gently touched to the drinking tube now present, and it was then released near the rear of the chamber. After the subject had completed a total of $55 \mathrm{sec}$ of contact with the drinking tube, it was returned to its home cage where water was again available. On the 3 rd day, the subjects were fitted with the ECS leads, placed in the chambers for 1 min., and the home cage water was removed. The next day, the subjects were again allowed to complete $55 \mathrm{sec}$ of contact with the drinking tube. The drinking tube was present in the chambers only during the two drink training days described above and on the test day described below.

On Day 5 the subjects were randomly assigned to one of four treatment conditions. Subjects in one group (TSFS/ECS) had the ECS leads attached and were placed separately into one of the two training-testing chambers. One minute later, the 15-sec tone was presented with strong footshock, delivered through the grid floor, occurring during the last $3 \mathrm{sec}$ of the tone. ECS followed $.5 \mathrm{sec}$ after offset of the tone and footshock. Subjects in two other groups were treated identically except that no ECS was delivered. One group (TSFS) received strong footshock paired with the tone, and the other group (TWFS) was given weak footshock paired with the tone. The weak footshock parameters were developed through pilot work to yield test performance similar to TSFS/ECS subjects. Subjects in the fourth group (NCSFS/ECS) were placed in the noncontingent treatment chamber and received strong footshock and ECS. This group served as control for any "systemic" effects of footshock and ECS.

Approximately half the subjects in each of the four major treatment groups received reminder treatment $96 \mathrm{~h}$ after training or noncontingent treatment. Reminder treatment consisted of the automatic presentation of the tone for $15 \mathrm{sec}$, beginning $1 \mathrm{~min}$ after placement in the drinking chamber. One hour later, these subjects were again removed from the home cage and given weak reminder footshock in the grid box. Previous studies (DeVietti \& Hopfer, 1974a,b) have shown this reminder procedure effective in producing recovery of memory in TSFS/ECS subjects. The remaining subjects in each of the four major groups were neither exposed to the drinking chamber and tone nor were they given weak reminder footshock. All subjects were tested, $24 \mathrm{~h}$ thirsty, the following day. In the test, the tone was automatically presented and remained on following an initial $50 \mathrm{sec}$ of contact with the drinking tube by the subject. Allowing $50 \mathrm{sec}$ of tube contact prior to the tone onset insured that subjects from all groups were at a comparable baseline prior to the presentation of the specific cue used to establish fear conditioning. The time (in seconds), transformed to logarithms, for the subjects to complete an additional $5 \mathrm{sec}$ of tube contact in the presence of the tone was automatically recorded and served as data for analysis. Subjects failing to achieve $5 \mathrm{sec}$ of tube contact, in the presence of the tone, within $600 \mathrm{sec}$ were removed, and a test score of $600(\log , 2.78)$ was recorded.

\section{Results}

Table 1 summarizes the treatment procedures and the test performance of the various groups. An overall 2 by 4 factorial analysis of variance with reminder (present or absent) and groups serving as main effects showed that both of these variables were reliable sources of variance $[F(1,90)=7.67, \quad p<.01 ; \quad F(3,90)=22.28, \quad p<.01$, respectively). However, the interaction between these sources did not approach statistical reliability. Specific $t$ tests using the error term from the overall analysis in the estimation of the standard error (Kirk, 1968) were made to compare performances in various specific groups. All differences that follow reached significance at least at the .05 level, two-tailed.

Overall, the $t$ tests revealed that reminder treatment induced memory recovery in both TSFS/ECS and TWFS groups. Specifically, the performance of subjects not given reminder indicated that TSFS/ECS and TWFS subjects had shorter drink latencies than TSFS subjects, thus demonstrating that both ECS following strong training and weak training alone resulted in less memory than controls given strong training and no amnesic treatment. Additionally, no statistical difference was discernable between TSFS/ECS and TWFS subjects although TWFS subjects had longer drink latencies than NCSFS/ECS subjects while this comparison was not significant in the case of TSFS/ECS subjects.

Reminder significantly increased drink latencies in both TSFS/ECS and TWFS subjects but had no effect in either TSFS or NCSFS/ECS subjects, as revealed by comparing the reminder groups to their no-reminder controls. As was the case in the comparison of groups given no reminder, no difference was obtained between TSFS/ECS and TWFS subjects after reminder. Moreover, the increase in test latencies in these two groups following reminder was substantial as neither group differed from the TSFS group not given reminder.

\section{Discussion}

Reminder influenced memory recovery in both TSFS/ECS and TWFS groups. This finding, obtained in a fear conditioning paradigm, is consistent with reports of 
this effect in passive avoidance tasks (Gold et al., 1973; Haycock et al., 1973). These latter investigators interpreted the parallel modification of retention in subjects given weak training only and subjects given strong training and amnesic treatment in terms of a generalization hypothesis which holds that reminder effected increased retention by the same mechanism in both groups. According to this view, the present data may have resulted from the interaction of reminder with a weak memory established by weak training and, in the case of TSFS/ECS subjects, a weak memory surviving the disruptive effects of ECS on memory storage. It should be noted, however, that parallel performance in these two groups simply indicates that, under a given set of conditions, reminder produced similar results. As such, this result cannot be considered as strong support for the notion that reminder increased retention in both groups by the same mechanism.

Another explanation of the present results, and, indeed, the results of Gold et al. (1973) and Haycock et al. (1973), is that reminder effected increased retention in strong trained subjects given amnesic treatment and weak trained subjects by different mechanisms. For example, increased retention in TWFS subjects could have resulted from the summation of two weak memories as suggested by Gold et al. (1973) and Haycock et al. (1973). On the other hand, increased retention in TSFS/ECS subjects could have resulted from the release of a strong but formerly unretrievable memory by reminder.

Differentiation of these two explanations of the reminder effect in amnesic subjects may be possible by the continued study of the effects of reminder in subjects given weak training only and in subjects given strong training and amnesic treatment in a variety of situations. The notion that reminder is effective by a mechanism common to both groups would be supported, but then only very weakly, by the continued finding of parallel modification of retention in both groups in all situations studied: Parallel modification by reminder could still result from different mechanisms in the two groups. On the other hand, the notion that reminder operates by different mechanisms in these groups would be supported by evidence of differential modification of performance following reminder.

\section{EXPERIMENT II}

The present experiment was designed to determine if reminder, shown to have similar effects in TSFS/ECS and TWFS in Experiment I, would continue to produce parallel results under slightly different conditions. In the present experiment, subjects were tested both prior to and following reminder footshock so that correlations could be calculated in order to determine if the magnitude of memory recovery is related to the degree of memory present prior to reminder. A significant direct relationship is predicted by the generalization hypothesis of reminder effects, and data have been obtained in support of this prediction in a passive avoidance task (Haycock et al., 1973). In addition, two levels of reminder footshock were employed in the present experiment.

\section{Method}

Subjects and apparatus. Subjects were 118 rats of the same age, history, and source described in Experiment I. Also, the same apparatus employed previously was used in the present experiment.

Procedure. The subjects were treated identically as in Experiment I through fear conditioning in which the four major treatment groups were again formed: TSFS/ECS, TSFS, TWFS, and NCSFS/ECS. All subjects were water deprived starting $72 \mathrm{~h}$ after training or noncontingent treatment. Approximately two-thirds of the subjects in each treatment group were tested the following day. Approximately half of these were given weak reminder footshock $(.6 \mathrm{~mA} ; 3 \mathrm{sec}) 1 \mathrm{~h}$ later as in Experiment $\mathrm{I}$. The other half received strong reminder footshock $(1.6 \mathrm{~mA}$; $3 \mathrm{sec}$ ). Subjects not tested in the first test were given $5 \mathrm{~min}$ of water access in the home cage. All subjects were given $2 \mathrm{~h}$ of access to water in the home cage $1 / 2 \mathrm{~h}$ after reminder footshock or at a comparable time in the case of control subjects not tested nor given reminder footshock. All subjects were tested, $24 \mathrm{~h}$ thirsty, the following day. Thus, approximately two-thirds of the subjects were tested before and following reminder while one-third were tested only once and served as controls for the reminder treatment. Initiation of two successive test days required that tested subjects and their controls be 24-h water deprived on 2 successive days. Pilot work (unpublished) with this severe water deprivation schedule indicated that it produced a significant decrease in drink latencies of TSFS and NCSFS/ECS subjects in the second test. The effect appears to be due to increased thirst rather than extinction or habituation since these groups did not differ significantly from similarly treated and water deprived subjects tested only in the second test. Thus, this producedure of two successive 24-h water deprivation days, besides providing information regarding the subjects' performance prior to reminder, greatly increases the tendency to drink in the second test. As such, this "titration" procedure provides a rigorous baseline with which to evaluate the response to reminder in TSFS/ECS and TWFS groups.

\section{Results}

Table 2 summarizes the treatment conditions and the test performance of the various groups. All reported differences are significant at least at the .05 level, two-tailed. Analysis of variance and subsequent $t$ tests of the first test performance (before reminder) of the four major groups indicated that TSFS/ECS and TWFS groups were closely matched prior to reminder (mean logs 1.99 and 1.98 , respectively) and that both groups showed intermediate latencies when compared to TSFS and NCSFS/ECS groups. Thus, both TSFS/ECS and TWFS resulted in weak memory prior to reminder. Random assignment to reminder conditions (strong or weak) was effective as there were no differences between subgroups prior to reminder footshock.

A split-plot 2 by 2 by 4 analysis of variance with level of reminder footshock (strong or weak) test (first and second), and groups serving as main effects, evaluated the change in performance of the repeatedly tested groups 
Table 2

Treatments and Retention Performance (Mean Log Drink Latencies \pm 1 Standard Error) of Groups in Experiment II

\begin{tabular}{|c|c|c|c|c|c|}
\hline \multirow{3}{*}{$\frac{\text { Group }}{\text { TSFS-SR }}$} & \multirow{3}{*}{$\frac{N}{11}$} & \multicolumn{2}{|c|}{ Reminder } & \multirow{2}{*}{\multicolumn{2}{|c|}{ Test 2}} \\
\hline & & $\begin{array}{c}\text { Test } 1 \\
\text { (Training Cues) }\end{array}$ & \multirow{2}{*}{$\frac{\text { Footshock }}{\text { Strong }}$} & & \\
\hline & & $2.54 \quad( \pm .07)$ & & 2.47 & $( \pm .10)$ \\
\hline TSFS-WR & 8 & $2.63 \quad( \pm .05)$ & Weak & 2.19 & $( \pm .15)$ \\
\hline TSFS-NR & 10 & None & None & 2.41 & $( \pm .08)$ \\
\hline TWFS-SR & 10 & $1.97( \pm .08)$ & Strong & 1.67 & $( \pm .11)$ \\
\hline TWFS-WR & 9 & $2.00 \quad( \pm .10)$ & Weak & 1.87 & $( \pm .17)$ \\
\hline TWFS-NR & 11 & None & None & 1.89 & $( \pm .11)$ \\
\hline TSFS/ECS-SR & 11 & $2.04 \quad( \pm .07)$ & Strong & 2.21 & $( \pm .12)$ \\
\hline TSFS/ECS-WR & 10 & $1.94( \pm .06)$ & Weak & 1.84 & $( \pm .11)$ \\
\hline TSFS/ECS-NR & 8 & None & None & 1.83 & $( \pm .13)$ \\
\hline NCSFS/ECS-SR & 10 & $1.82( \pm .09)$ & Strong & 1.42 & $( \pm .14)$ \\
\hline NCSFS/ECS-WR & 11 & $1.81 \quad( \pm .06)$ & Weak & 1.39 & $( \pm .11)$ \\
\hline NCSFS/ECS-NR & 9 & None & None & 1.55 & $( \pm .11)$ \\
\hline
\end{tabular}

Note-Description of the treatment groups may be found in the text. Abbreviations of reminder conditions are: SR = strong reminder, $W R=$ weak reminder, $N R=$ no reminder.

between the first and second tests, i.e., before and after reminder. The main effects of groups, level of reminder, and test were reliable sources of variance. In addition, the interaction between groups and tests was also reliable. No other source of variance approached statistical reliability.

The analysis of the breakdown of the interaction between groups and tests provided some evidence for a differential effect of reminder in TSFS/ECS and TWFS groups. All major groups, TSFS, TWFS, and NCSFS/ECS, with the notable exception of the TSFS/ECS group, showed significant decreased latencies in the second test, relative to the first; whereas the TSFS/ECS group showed a nonsignificant increase in the second test.

Specific $t$ tests of subgroups' performance in the second test, i.e., following reminder, provided strong evidence of a differential effect of reminder between TSFS/ECS and TWFS groups. Following strong reminder, TSFS/ECS subjects had significantly longer drink latencies than TWFS subjects. There was no statistical difference obtained in comparisons of the TSFS/ECS groups to the TSFS group or in comparison of the TWFS to the NCSFS/ECS group. Thus, reminder was apparently specific to TSFS/ECS subjects and had no "reminder" effect in TWFS subjects.

The comparisons in subjects given weak reminder footshock indicated that weak reminder did not result in differential performance between TSFS/ECS and TWFS subjects as in Experiment I, but this parallel effect was manifest in the present experiment as a nonsignificant decrease in latencies in the second test.

A 3 by 4 factorial analysis of variance, with reminder (strong, weak, or none) and the four major treatment groups as main effects, evaluated drink latencies in the second test. The main effect of groups reached statistical reliability, but neither reminder nor the interaction of groups and reminder approached significance. The test performance of subjects in each reminder condition (strong or weak) was compared to their no-reminder controls in each of the four major groups with $t$ tests. These evaluations indicated that the significant decrease in drink latencies in the second test observed in the split-plot analysis for the TSFS, TWFS, and NCSFS/ECS groups probably could be attributed to increased thirst, as none of the comparisons between these groups to their no-reminder controls reached statistical significance. On the other hand, the TSFS/ECS group given strong reminder showed significantly longer drink latencies than its control, thus demonstrating memory recovery in spite of the rigorous baseline provided by the water deprivation schedule.

Finally, correlations (Pearson r) were calculated using the test data in the first and second tests for TSFS/ECS and TWFS groups. In TSFS/ECS subjects given strong and weak reminder footshock, the correlations were -.05 and -.15 , respectively. In TWFS subjects, the same evaluations yielded correlations of -.10 and .21 . None of these correlations approached statistical reliability.

\section{Discussion}

The results of the present experiment provide evidence of a differential effect of reminder in subjects given strong training and rendered amnesic and in subjects given weak training alone.

In the weak reminder condition, as in Experiment I, reminder resulted in a parallel modification of test performance in TSFS/ECS and TWFS subjects. This finding corroborates the findings of others (Gold et al., 1973; Haycock et al., 1973) and lends some limited support to the notion that reminder provides a weak training experience which generalizes to and summates with a memory remnant surviving ECS. However, the additional finding in the present experiment that strong reminder occasioned memory recovery in TSFS/ECS subjects while having no measurable "reminder" effect 
in TWFS subjects is difficult to assimilate into this generalization position. If ECS allowed consolidation of but a weak memory, reminder should have resulted in the same effect in both TSFS/ECS and TWFS groups. Clearly this was not the case in the strong reminder condition. This finding would seem more consistent with the notion that reminder releases for behavioral expression a formerly strong memory originally suppressed by ECS. In turn, these data suggest that the major effect of ECS is on memory retrieval processes. Also, the results of the correlations determined between the test performance before and after reminder in ECS subjects failed to provide any support for the generalization hypothesis. Although the sample sizes were small and the correlations not significant, the relationship appeared to be indirect rather than direct, as predicted by the generalization hypothesis.

The present results, in addition to a recent report showing reminder to be effective when administered to ECS subjects evidencing strong amnesia and reminder to be ineffective in subjects showing weak amnesia (DeVietti \& Bucy, 1975), a result directly contrary to the generalization hypothesis predictions, would seem to call for a reevaluation of this hypothesis.

Further use of techniques designed to test for differential effects of reminder in subjects given strong training and ECS and subjects given weak training may prove to be a valuable approach in determining the mechanism by which reminder influences memory recovery in amnesic subjects.

\section{REFERENCES}

Cherkin, A. Retrograde amnesia in the chick: Resistance to the reminder effect. Phy siology and Behavior, 1972, 8, 949-955.
DeVietti, T. L., \& Bucy, C. E. Recovery of memory after reminder: Evidence for two forms of retrieval deficit induced by ECS. Phy siological Psy chology, 1975, 3, 19-25.

DeVietti, T. L., \& Hopfer, T. J. Complete amnesia induced by ECS and complete recovery of memory following reinstatement treatment. Physiology and Behavior, 1974, 12, 599-603. (a)

DeVietti, T. L., \& Hopfer, T. J. Reinstatement of memory in rats: Dependence upon two forms of retrieval deficit following ECS. Journal of Comparative and Physiological Psychology, 1974, 86, 1090-1099. (b)

DeVietti, T. L., \& Larson, R. C. ECS effects: Evidence supporting state-dependent learning in rats. Journal of Comparative and Physiological Psychology, 1971, 74, 407-415.

Gold, P. E., Haycock, J. W., Macri, J., \& McGaugh, J. L. Retrograde amnesia and the "reminder effect": An alternative interpretation. Science, 1973, 180, 1199-1201.

Gold, P. E., \& King, R. A. Retrograde amnesia: Storage failure versus retrieval failure. Psychological Review, 1974, 81, 465-469.

Hay cock, J. W., Gold, P. E., Macri, J., \& McGaugh, J. L. Noncontingent footshock attenuation of retrograde amnesia: A generalization effect. Phy siology and Behavior, 1973, 11, 99-102.

Hayes, K. J. Cognitive and emotional effects of electroconvulsive shock in rats. Joumal of Comparative and Physiological Psy chology, 1948, 41, 40-61.

Kesner, R. P., \& Conner, H. S. Cue-dependent recovery from ECS-induced amnesia: Evidence for time dependence. Phy siological Psychology, 1974, 2, 123-125.

Kirk, R. E. Experimental design: Procedures for the behavioral sciences. Belmont, California: Wadsworth, 1968.

Koppenaal, R. J., Jagoda, E., \& Cruce, J. A. F. Recovery from ECS-produced amnesia following a reminder. Psychonomic Science, 1967, 9, 293-294.

Lewis, D. J. Sources of experimental amnesia. Psychological Review, 1969, 76, 461-472.

Lewis, D. J., Misanin, J. R., \& Miller, R. R. Recovery of memory following amnesia. Nature, 1968, 220, 704-705.

Mah, C. J., \& Albert, D. J. Electroconvulsive shock-induced retrograde amnesia: An analysis of the variation in the length of the amnesia gradient. Behavioral Biology, 1973, 9, 517-540.

Miller, R. R., \& Springer, A. D. Amnesia, consolidation and retrieval. Psychological Review, 1973, 80, 69-79.

Miller, R. R., \& Springer, A. D. Implications of recovery from experimental amnesia. Psychological Review, 1974, 81, 470-473.

(Received for publication November 11, 1974; revision accepted February 20, 1975.) 